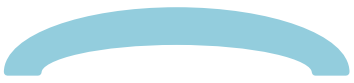

\title{
Anadolu Popülasyonunda Talus ile Calcaneus'un Morfometrik Özellikleri ve Ortak Eklem Yüzlerinin Tiplendirilmesi
}

\section{Morphometric Features of Talus and Calcaneus in The Anatolian Population and Typing of Common Joint Faces}

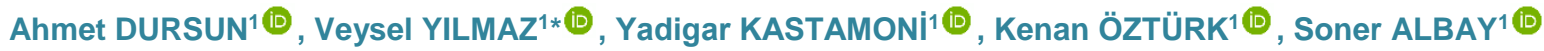

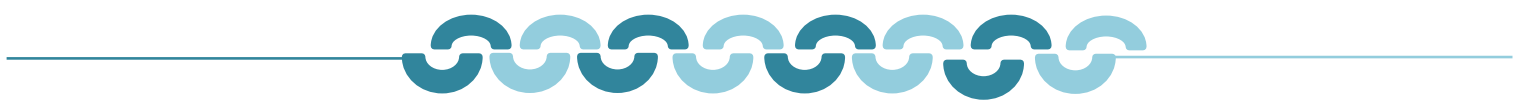

\section{ÖZET}

Amaç: Çalışmamızda Anadolu popülasyonuna ait talus ve calcaneusların morfometrik ölçümlerini yapıp, birlikte oluşturmuş oldukları articulatio subtalaris ve articulatio talocalcaneonavicularis'e katılan eklem yüzlerini sınıflandırarak antropometrik index oluşumuna katkıda bulunmak amaçlanmıştır.

Materyal-Metot: Anatomi Ana Bilim Dalı laboratuvarında mevcut olan kemiklerden, yaş ve cinsiyete bakılmaksızın toplamda 18 adet calcaneus ve 23 adet talus değerlendirildi. Calcaneus ve talus'a ait morfometrik ölçümler alındı. Ölçümlerde dijital kumpas kullanıldı. Talus ve calcaneus'un birbirleri ile olan eklem yüzleri Bunning ve Barnett'in yapmış olduğu tiplendirmeye göre A, B ve C tipleri ve bu tiplere ait alt tipler olmak üzere sınıflandırıldı.

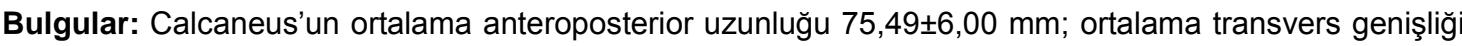
$39,31 \pm 3,68 \mathrm{~mm}$; ortalama Böhler açısı $29,23^{\circ} \pm 6,80^{\circ}$; ortalama sulcus calcanei genişliği $5,72 \pm 1,73 \mathrm{~mm}$; ortalama sulcus calcanei uzunluğu $22,46 \pm 4,22 \mathrm{~mm}$ olarak ölçüldü. Talus'un ortalama anteroposterior uzunluğu $55,34 \pm 5,49 \mathrm{~mm}$; ortalama transvers genişliği $41,22 \pm 3,84 \mathrm{~mm}$; ortalama sulcus tali genişliği

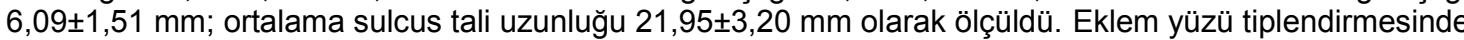
ise talus ve calcaneus'un eklem yüzlerinin büyük oranda Tip B olduğu belirlenmiştir.

Sonuç: Talus ve calcaneus'un morfometrik ölçüleri ve birbirleri ile olan eklem yüzlerinin tipleri popülasyonlar arası farklııık göstermektedir. Çalışmamızda, bazı toplumlarla benzer şekilde talus ve calcaneus eklem yüzü tiplendirilmesinde Tip B yaygın olarak bulunmuş ve popülasyonlar arası karşılaştırmalar yapılmıştır. Talus ve calcaneusa ait morfometrik özelliklerin ve popülasyonlar arası farklılıkların bilinmesi, bu eklemleri içeren birçok patolojinin tedavi yönteminin belirlenmesinde oldukça önemlidir.

Anahtar Kelimeler: Böhler açısı, articulatio talocalcaneonavicularis, subtalar eklem, articulatio talocalcanea

Alınış / Received: 03.07.2021 Kabul / Accepted: 21.09.2021 Online Yayınlanma / Published Online: 20.12.2021 


\title{
$\cos 2020$
}

\begin{abstract}
Objective: In our study, it was aimed to contribute to the formation of anthropometric index by making morphometric measurements of talus and calcaneus belonging to the Anatolian population and classifying the articulatio subtalaris and articulatio talocalcaneonavicularis that they formed together.
\end{abstract}

Material-Method: A total of 18 calcaneus and 23 talus were evaluated from the bones available in the Anatomy Department laboratory, regardless of age and gender. Digital calipers were used in the measurements. The joint faces of talus and calcaneus with each other were classified as Bunning and Barnett.

Results: The average anteroposterior length of Calcaneus was $75.49 \pm 6.00 \mathrm{~mm}$; mean transverse width $39.31 \pm 3.68 \mathrm{~mm}$; average Böhler angle $29.23^{\circ} \pm 6.80^{\circ}$ average sulcus calcanei width $5.72 \pm 1.73 \mathrm{~mm}$; The average sulcus calcanei length was measured as $22.46 \pm 4.22 \mathrm{~mm}$. The average anteroposterior length of the talus is $55.34 \pm 5.49 \mathrm{~mm}$; mean transverse width $41.22 \pm 3.84 \mathrm{~mm}$; average sulcus tali width $6.09 \pm 1.51 \mathrm{~mm}$; The average sulcus tali length was measured as $21.95 \pm 3.20 \mathrm{~mm}$. In the joint face typing, it has been determined that the joint faces of the talus and calcaneus are mostly Type B.

Conclusion: The morphometric measurements of talus and calcaneus and the types of joint faces with each other differ between populations. In our study, similar to some populations, type B was found commonly in talus and calcaneus joint face typing and comparisons between populations were made. Knowing the morphometric properties of talus and calcaneus and the differences between populations is very important in determining the treatment method of many pathologies involving these joints.

Keywords: Böhler angle, talocalcaneonavicular joint, subtalar joint, talocalcaneal joint

\section{Giriş}

Tarsal kemikler; tibia, fibula ve metatarsal kemiklerin arasında bulunurlar. Calcaneus en büyük tarsal kemiktir ve üzerinde bulunan talus ile articulatio subtalaris (articulatio talocalcanea) ve articulatio talocalcaneonavicularis'i oluşturur. Bu eklemler ise inversiyon ve eversiyon hareketlerini gerçekleştirirler.

Topuk kemiği olan calcaneus kuvvetin aktarılmasında önemli rol oynar ve bacağın arka tarafındaki yüzeyel fleksör kaslar için de bir kaldıraç kolu niteliği taşımaktadır. Calcaneus'un üst yüzü ön ve arka olarak iki bölüme ayrılmıştır. Ön bölümde üç adet eklem yüzü bulunur. Arkada bulunan facies articularis talaris posterior diğer ikisinden daha büyüktür ve sulcus calcanei ile diğer ikisinden ayrılır. Sulcus calcanei'nin hemen önünde, sustentaculum tali üzerinde bulunan eklem yüzüne facies articularis talaris media denir. Bunun da ön kısmında bulunan eklem yüzü ise facies articularis talaris anterior'dur.

Talus, tarsal kemikler arasında calcaneus'tan sonraki en büyük kemiktir. Tarsal kemiklerin en proksimalinde olması sebebiyle bacak kemikleri ile tarsal kemikler arasında bağlantı kuran tek kemiktir. Hiçbir kasın tutunmadığı ancak çok sayıda ligamentin tutunduğu talus, caput tali, collum tali ve corpus tali olarak üç bölümde incelenmektedir. Corpus tali'nin alt yüzünde bulunan sulcus tali ismi verilen bir oluk ile birbirinden ayrılmış eklem yüzleri bulunur. Bu eklem yüzlerinden sulcus tali'nin arkasında bulunana facies articularis calcanea posterior, sulcus tali'nin önünde bulunanlara ise facies articularis calcanea media ve facies articularis calcanea anterior denilir. Facies articularis calcanea posterior diğerlerinden daha büyüktür ve calcaneus'un üst yüzünün arka tarafında bulunan facies 
articularis talaris posterior ile art. subtalaris'i oluşturur. Facies articularis calcanea media calcaneus'un sustentaculum tali'si üzerindeki facies articularis talaris media ile caput tali'nin ön alt kısmındaki facies articularis calcanea anterior ise calcaneus'daki facies articularis talaris anterior ile art. talocalcaneonavicularis'in bir kısmını oluştururlar [1].

Genelde art. subtalaris ve art. talocalcaneonavicularis'e ait 3 adet eklem yüzü bulunmaktadır [1]. Ancak bu eklem yüzü sayıları ve şekilleri toplumsal ve bireysel farklııklar göstermektedir. Bu farklılıklar ise ayaktaki statik ve kinetik dinamik üzerinde önemli rol oynamaktadır. Bazı araştırmacılar, ayrılma derecesi, füzyon ve şekil gibi parametreleri kullanarak eklem yüzeylerinin tiplendirmesini yapmışlardır [2]. Klinik tedavilerde, hekim tarafından talus ve calcaneus'un birlikte yapmış oldukları eklem yüzlerine ait toplumsal ve bireysel farklılıklar gösteren yaygın varyasyonel durumların, pes planus, pes ekinovarus, talocalcaneal artrit, talocalcaneal koalisyon, eklemi içeren kırıklar, valgus deformiteleri, subtalar instabilite ve subtalar implant uygulamaları gibi durumlarda tedavi sonuçlarının iyi olması için bilinmesi gerekmektedir [2].

Calcaneus en sık yaralanan tarsal kemiktir ve tüm kırıkların \%2'sini oluşturur. Tüm tarsal kemik kırıklarının \%65'i calcaneus kırıklarıdır. Calcaneus kırıklarının ise \%75'i intraartiküler kırıklardır. Bu intraartiküler kırıklar talus'u da direk olarak etkilemektedir. Bu kırıklarının tedavisinde calcaneus ve talus'un anatomik özelliklerinin doğru şekilde anlaşılması, uygulanacak tedavinin başarılı sonuçlanmasında oldukça önemlidir [3]. Ayrıca calcaneus kırıklarını değerlendirmede Böhler açıSı da kullanılmaktadır. Böhler açısı: Calcaneus'un facies articularis talaris posterioru'nun en yüksek noktası ile tuber calcanei'nin en yüksek noktasını birleştiren çizgi ve facies articularis talaris posterior'un en yüksek noktası ile calcaneus'un en anterosuperior noktasını birleştiren çizgi arasında kalan açıdır [3]. Böhler'in normal açı değerleri $25^{\circ}-40^{\circ}$ arasında değişmektedir. Ameliyat öncesi azalmış Böhler açısı kırık ameliyatı için hasta lehinedir [4].

Articulatio subtalaris'e katılan eklem yüzlerinin varyasyonel morfolojisi bu eklemin hareket açıklığını etkileyebilir ve bu eklemin instabilitesine, eklem bağlarının gevşemesine ve eklemde artrite yol açabilir [5]. Talus veya calcaneus'taki morfolojik değişikliklerin en ufak bir kısmı bile bu eklemlerin biyomekaniği üzerinde olumsuz bir etki yaratabilir. Bu sebeple talus ve calcaneus'un genel morfometrik özellikleri ve bu eklemlere katılan eklem yüzü tipleri bilinmelidir.

Pes planus, talocalcaneal artrit, talocalcaneal koalisyon, eklem içi kırıklar, doğuştan dismorfoloji, valgus deformiteleri, gibi birçok konjenital ya da konjenital olmayan ayak deformitelerinde uygulanan tedavi seçenekleri de yumuşak dokuların gevşetilmesiyle birlikte osteotomi ve anatomik redüksiyondur [6]. Bu gibi patolojik durumlarda talus ve calcaneusa ait morfolojik özelliklerin yapısal ve toplumsal farklarının iyi anlaşılması, radyologların subtalar bölgedeki patolojiyi ve anatomik varyasyonları tanımlamasında, cerrahi hekimlerin ise bu bölgede yapacağı yapısal temelli cerrahi prosedürleri belirlenmesinde gereklidir.

\section{Materyal ve Metot}

Tıp Fakültesi Anatomi Ana Bilim Dalı laboratuvarında mevcut olan kemiklerden, yaş ve cinsiyete bakılmaksızın toplamda 18 adet calcaneus ( 7 sol, 11 sağ) ve 23 adet talus (12 sol, 11 sağ) değerlendirildi. Çalışma için Süleyman Demirel Üniversitesi Tıp Fakültesi Klinik Araştırmalar Etik Kurulu'ndan onay alındı. Bu çalışmada; Böhler açısı metal gonyometre kullanılarak, diğer parametreler ise dijital kaliper (BTS 12044) kullanılarak ölçülmüştür.

Talus ve calcaneus'un üzerindeki ortak eklem yüzeyleri Bunning \& Barnett'in yapmış olduğu sınıflandırma kullanılarak aşağıdaki şekilde tiplendirildi [7]. Calcaneus için Tip A'da facies articularis talaris anterior, facies articularis talaris media ve facies articularis talaris posterior ayrı eklem yüzleri olarak görülmektedir. Facies articularis talaris anterior ve media arasındaki mesafeye ve eklem yüzü şekillerine göre Tip A'nın üç alt tipi vardır. Tip A1, facies articularis talaris anterior ve media arasındaki mesafe 2 mm'den küçüktür; Tip A2, facies articularis talaris anterior ve media arasındaki mesafe 2-5 mm'dir. Tip A3, facies articularis talaris anterior ve media arası mesafe $5 \mathrm{~mm}$ 'den fazladır (Şekil $1 \mathrm{~A}$ ).

Tip B'de facies articularis talaris anterior ve facies articularis talaris media arasında ayrılma yoktur ve iki alt tipe ayrılmıştır. Birincisi iki eklem yüzünün oluşturduğu birleşim hattının belirgin olduğu Tip B1'dir. İkincisi ise facies articularis talaris anterior ve media arasında ayrımın görülmediği, tamamen 
tek bir eklem gibi görünen Tip B2'dir. Tip C'de ise facies articularis talaris anterior, media ve posterior arasında ayrım yoktur ve birleşmiş tek bir eklem yüzü şeklinde görülür [6] (Şekil 1B).

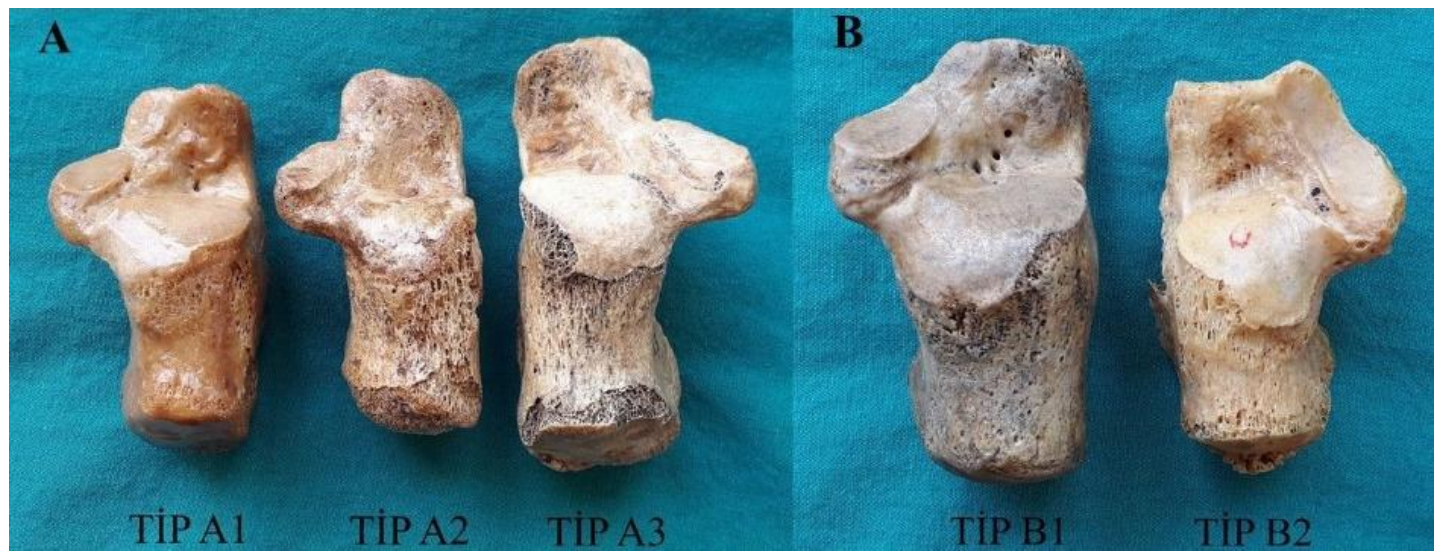

Şekil 1: Calcaneus'a ait mevcut eklem yüzlerinin tiplendirilmesi

Ayrıca Calcaneus'a ait aşağıdaki parametreler ölçüldü.

1) Calcaneus'un anteroposterior uzunluğu (CAPU); ön yüzeydeki en ön nokta ile arka yüzeydeki en arka nokta arasındaki mesafe olarak ölçüldü.

2) Calcaneus'un transvers genişliği (CTG); medial yüzeydeki en medial nokta ile lateral yüzeydeki en lateral nokta arasındaki mesafe olarak ölçüldü.

3) Sulcus calcanei uzunluğu (SCU); sulcus calcaneinin ön ve arka kenarları arasındaki mesafe olarak ölçüldü.

4) Sulcus calcanei genişliği (SCG); sulcus calcaneinin medial kenarı ile lateral kenarı arasındaki mesafe olarak ölçüldü (Şekil 2).

5) Böhler açısı; gonyometre kullanarak ölçüldü (6) (Şekil 3).

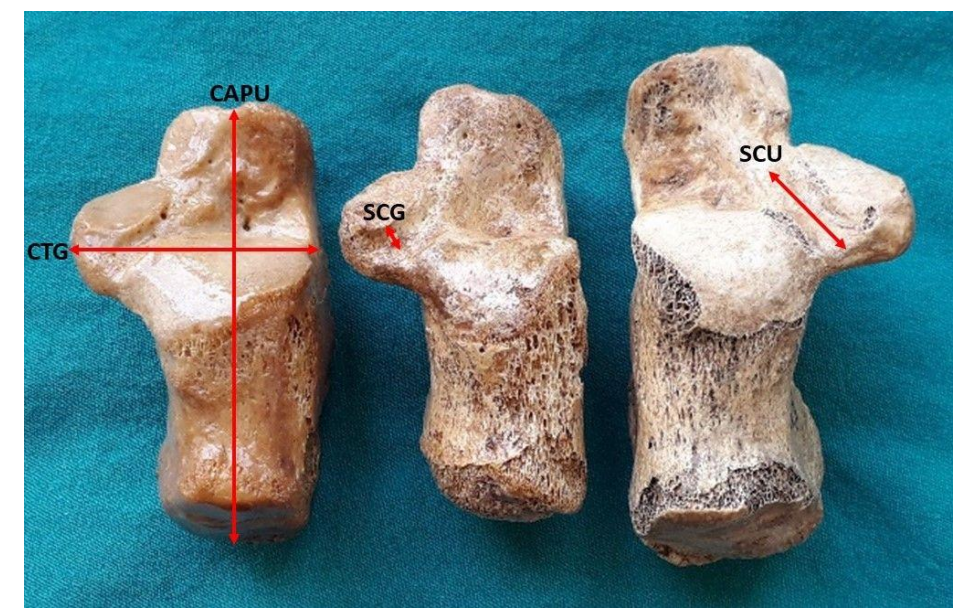

Şekil 2: Calcaneus'un morfometrik ölçümlerinin gösterilmesi. CAPU: Calcaneus'a ait anteroposterior uzunluk, CTG: Calcaneus'a ait transvers genişlik, SCU: Sulcus calcanei uzunluğu, SCG: Sulcus calcanei genişliği 


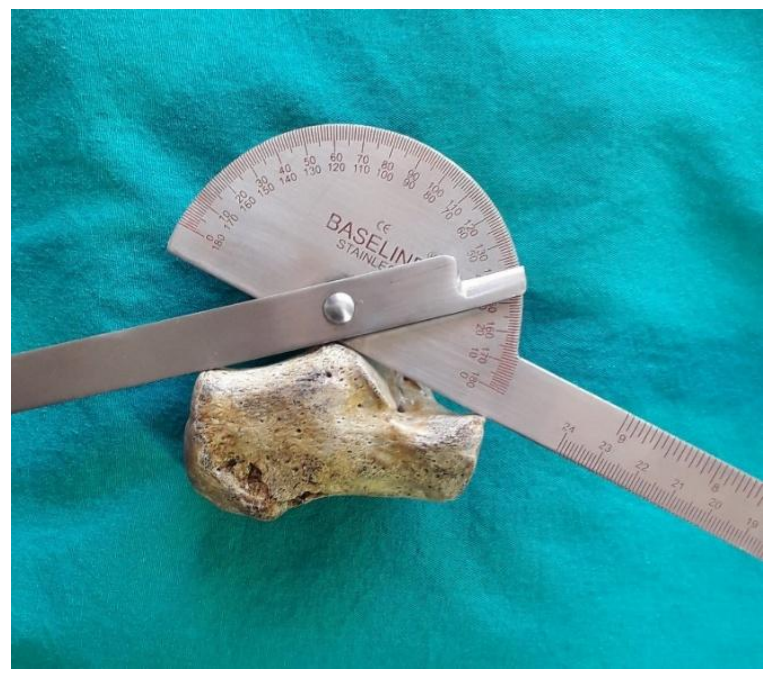

Şekil 3: Böhler açısı ölçümünün gösterilmesi

Talus için Tip A'da facies articularis calcanea anterior ve facies articularis calcanea media ve facies articularis calcanea posterior ayrı eklem yüzleri olarak görülmektedir. Facies articularis calcanea anterior ve media arasındaki mesafeye ve eklem yüzü şekillerine göre Tip A'nın üç alt tipi vardır. Tip A1, facies articularis calcanea anterior ve media arasındaki mesafe 2 mm'den küçüktür; Tip A2, facies articularis calcanea anterior ve media arasındaki mesafe 2-5 mm'dir. Tip A3, facies articularis calcanea anterior ve media arası mesafe $5 \mathrm{~mm}$ 'den fazladır. Tip B'de facies articularis calcanea anterior ve facies articularis calcanea media arasında ayrılma yoktur ve iki alt tipe ayrılmıştır. Birincisi iki eklem yüzünün oluşturduğu birleşim hattının belirgin olduğu Tip B1'dir. İkincisi ise facies articularis calcanea anterior ve media arasında ayrımın görülmediği, tamamen tek bir eklem gibi görünen Tip B2'dir. Tip C'de ise facies articularis calcanea anterior, media ve posterior arasında ayrım yoktur ve birleşmiş tek bir eklem yüzü şeklinde görülür [6] (Şekil 4).

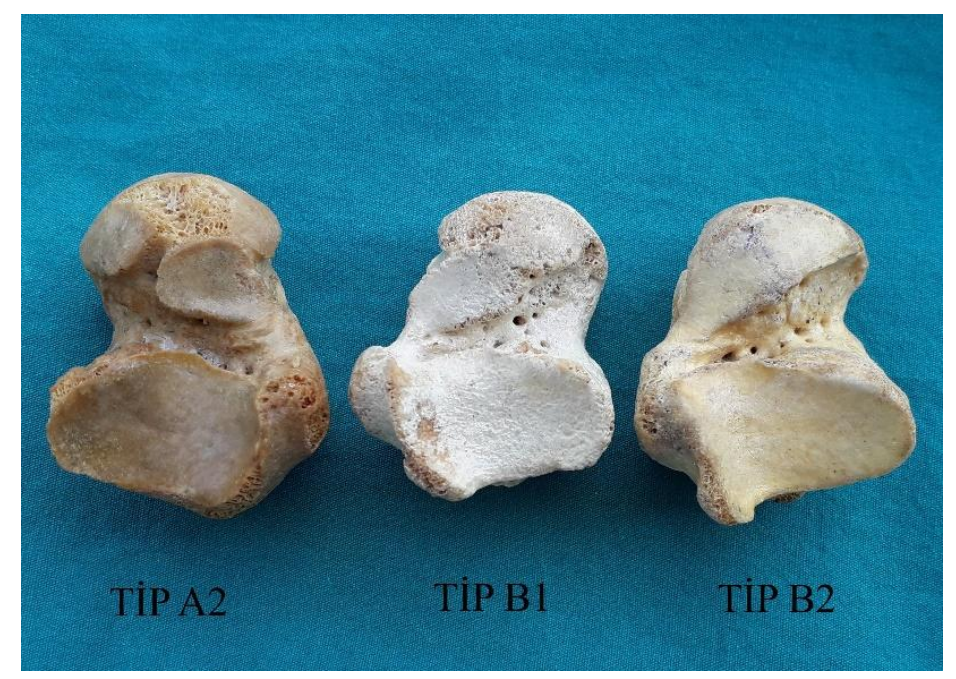

Şekil 4: Talus'a ait mevcut eklem yüzlerinin tiplendirilmesi

Ayrıca Talus'a ait aşağıdaki parametreler ölçüldü.

1) Talus'un anteroposterior uzunluğu (TAPU); ön yüzeydeki en ön nokta ile arka yüzeydeki en arka nokta arasındaki mesafe olarak ölçüldü.

2) Talus'un transvers genişliği (TTG); medial yüzeydeki en medial nokta ile lateral yüzeydeki en lateral nokta arasındaki mesafe olarak ölçüldü.

3) Sulcus tali uzunluğu (STU); sulcus tali'nin en medial ucu ile en lateral ucu arasındaki mesafe olarak ölçüldü.

4) Sulcus tali genişliği (STG); sulcus tali'nin ön kenarı ile arka kenarı arasındaki mesafe olarak ölçüldü [6] (Şekil 5). 


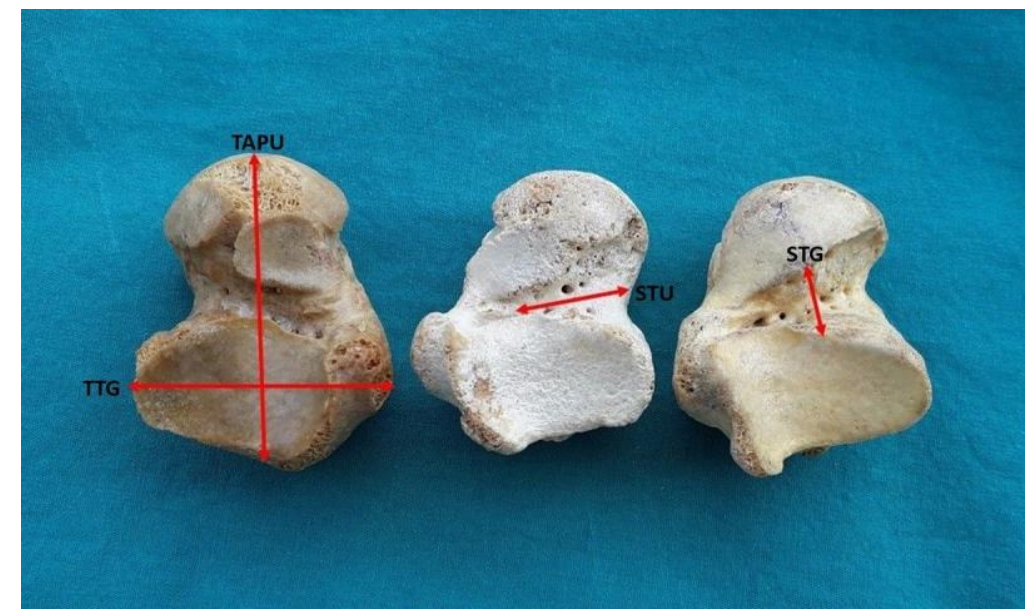

Şekil 5: Talus'un morfometrik ölçümlerinin gösterilmesi. TAPU: Talus'a ait anteroposterior uzunluk, TTG: Talus'a ait transvers genişlik, STU: Sulcus tali uzunluğu, STG: Sulcus tali genişliği

İstatiksiksel analizler SPSS (Versiyon 20.0, Armonk; NY, USA) programı kullanılarak gerçekleştirildi. İlgili parametrelerin sağ ve sol taraflara göre maksimum-minimum değerleri, aritmetik ortalamaları ve standart sapmaları belirlendi. Verilerimizin normal dağılıp dağılmadığını bulmak için normalite testleri (Kolmogorov Smirnov, Shapiro-wilk W, histogram grafiği, Q-Q plot) uygulanmıştır. Yapılan bu testlerin sonucunda verilerimizin tamamı normal dağılım gösterdiği için karşılaştırma analizinde parametrik testlerden independent samples $T$ testi kullanıldı. İstatistiksel analizlerde anlamlılık düzeyi $p<0,05$ olarak alındı.

\section{Bulgular}

Calcaneus ve Talus'a ait eklem yüzlerinin tiplendirilmesi tablo 1'de özetlenmiştir. Calcaneus'ta Tip A oranı \%27,6 (\%5,5 Tip A1; \%16,6 Tip A2; \%5,5 Tip A3), Tip B oranı \%72,1 (\%27,7 Tip B1; \%44,4 Tip B2) olarak bulunmuş ve Tip C'ye rastlanmamıştır. Talus'ta Tip A oranı \%8,6 (Tip A2), Tip B oranı \%91,3 (\%17,3Tip B1; \%73,9 Tip B2) olarak bulunmuş ve Tip C'ye rastlanmamıştır.

Tablo 1: Talus ve Calcaneus'ta gözlenen eklem yüzü tiplerinin sayısı ve yüzdesi

\begin{tabular}{ccccc}
\hline & \multicolumn{2}{c}{ Talus $(\mathbf{n = 2 3})$} & \multicolumn{2}{c}{ Calcaneus $(\mathbf{n = 1 8})$} \\
\hline Tip A1 & (n) & $(\%)$ & $(\mathbf{n})$ & $(\%)$ \\
Tip A2 & 0 & 0 & 1 & 5,5 \\
Tip A3 & 2 & 8,6 & 3 & 16,6 \\
Tip B1 & 0 & 0 & 1 & 5,5 \\
Tip B2 & 4 & 17,3 & 5 & 27,7 \\
Tip C & 17 & 73,9 & 8 & 44,4 \\
Toplam & 0 & 0 & 0 & 0 \\
\hline
\end{tabular}

Calcaneus'un ortalama anteroposterior uzunluğu sağ tarafta $74,87 \pm 5,26 \mathrm{~mm}$, sol tarafta $76,46 \pm 7,36$ $\mathrm{mm}$, ortalama transvers genişliği sağ tarafta $39,15 \pm 4,13 \mathrm{~mm}$, sol tarafta $39,57 \pm 3,16 \mathrm{~mm}$ olarak bulundu ve taraflar arasında fark yoktu. Ortalama sulcus calcanei uzunluğu sağ tarafta $23,00 \pm 4,11$ $\mathrm{mm}$, sol tarafta $21.62 \pm 4,56 \mathrm{~mm}$, ortalama sulcus calcanei genişliği sağda $6,05 \pm 1,90 \mathrm{~mm}$, solda $5,20 \pm 1,41 \mathrm{~mm}$ idi ve taraflar arasında anlamlı fark yoktu (Tablo 2). 
Tablo 2: Calcaneus'a ait morfometrik parametreler ve Böhler açısı'nın taraflara göre ortalama değerleri ve karşılaştırılması

\begin{tabular}{|c|c|c|c|c|}
\hline Parametreler & Taraf & Adet & $\begin{array}{c}\text { Ortalama } \pm \text { Std. } \\
\text { Sapma }\end{array}$ & $\mathbf{P}$ \\
\hline \multirow{3}{*}{ CAPU (mm) } & Sağ & 11 & $74,87 \pm 5,26$ & \multirow{3}{*}{,358 } \\
\hline & Sol & 7 & $76,46 \pm 7,36$ & \\
\hline & Toplam & 18 & $75,49 \pm 6,00$ & \\
\hline \multirow{3}{*}{ CTG (mm) } & Sağ & 11 & $39,15 \pm 4,13$ & \multirow{3}{*}{,360 } \\
\hline & Sol & 7 & $39,57 \pm 3,16$ & \\
\hline & Toplam & 18 & $39,31 \pm 3,68$ & \\
\hline \multirow{3}{*}{ Böhler açısı ( $\left.{ }^{\circ}\right)$} & Sağ & 11 & $30,18 \pm 7,45$ & \multirow{3}{*}{, 533} \\
\hline & Sol & 6 & $27,50 \pm 5,61$ & \\
\hline & Toplam & 17 & $29,23 \pm 6,80$ & \\
\hline \multirow{3}{*}{ SCG (mm) } & Sağ & 11 & $6,05 \pm 1,90$ & \multirow{3}{*}{,366 } \\
\hline & Sol & 7 & $5,20 \pm 1,41$ & \\
\hline & Toplam & 18 & $5,72 \pm 1,73$ & \\
\hline \multirow{3}{*}{$\mathrm{SCU}(\mathrm{mm})$} & Sağ & 11 & $23,00 \pm 4,11$ & \multirow{3}{*}{,868 } \\
\hline & Sol & 7 & $21,62 \pm 4,56$ & \\
\hline & Toplam & 18 & $22,46 \pm 4,22$ & \\
\hline
\end{tabular}

Talus'un ortalama anteroposterior uzunluğu sağ tarafta $55,25 \pm 5,59 \mathrm{~mm}$, sol tarafta $55,43 \pm 5,40 \mathrm{~mm}$, transvers genişliği sağ tarafta ortalama $40,96 \pm 3,73 \mathrm{~mm}$, sol tarafta $41,49 \pm 3,95 \mathrm{~mm}$ olarak bulundu ve taraflar arasında fark yoktu. Sulcus tali uzunluğu sağ tarafta ortalama $21,77 \pm 3,11 \mathrm{~mm}$, sol tarafta $22,14 \pm 3,29 \mathrm{~mm}$, sulcus tali genişliği sağ tarafta ortalama $5,87 \pm 1,38 \mathrm{~mm}$, sol tarafta $6,32 \pm 1,64 \mathrm{~mm}$ olarak bulundu ve taraflar arasında fark yoktu (Tablo 3 ). 
Tablo 3: Talus'a ait morfometrik parametrelerin taraflara göre ortalama değerleri ve karşılaştırılması

\begin{tabular}{|c|c|c|c|c|}
\hline Parametreler & Taraf & Adet & $\begin{array}{l}\text { Ortalama } \pm \text { Std. } \\
\text { Sapma }\end{array}$ & $\mathbf{P}$ \\
\hline \multirow{3}{*}{ TAPU (mm) } & Sağ & 9 & $55,25 \pm 5,59$ & \multirow{3}{*}{,576 } \\
\hline & Sol & 11 & $55,43 \pm 5,40$ & \\
\hline & Toplam & 20 & $55,34 \pm 5,49$ & \\
\hline \multirow{3}{*}{ TTG (mm) } & Sağ & 9 & $40,96 \pm 3,73$ & \multirow{3}{*}{,929 } \\
\hline & Sol & 11 & $41,49 \pm 3,95$ & \\
\hline & Toplam & 20 & $41,22 \pm 3,84$ & \\
\hline \multirow{3}{*}{ STG (mm) } & Sağ & 11 & $5,87 \pm 1,38$ & \multirow{3}{*}{,629 } \\
\hline & Sol & 12 & $6,32 \pm 1,64$ & \\
\hline & Toplam & 23 & $6,09 \pm 1,51$ & \\
\hline \multirow{3}{*}{ STU (mm) } & Sağ & 11 & $21,77 \pm 3,11$ & \multirow[t]{3}{*}{,320 } \\
\hline & Sol & 12 & $22,14 \pm 3,29$ & \\
\hline & Toplam & 23 & $21,95 \pm 3,20$ & \\
\hline
\end{tabular}

\section{Tartışma ve Sonuç}

Çalışmamızda, art. subtalaris ve art. talocalcaneonavicularis'te talus'a ait eklem yüzü tiplerinin $\% 91,3$ oranında Tip B (\%17,3 Tip B1, \%73,9 Tip B2), \%8,6'sının Tip A2 olduğu, calcaneus'un \%72,2 oranında Tip B (\%27,7 Tip B1, \%44,4 Tip B2), \%27,7'sinin ise Tip A (\%5,5 Tip A1, \%16,6 Tip A2, \%5,5 Tip A3) olduğu belirlenmiştir (Tablo 1). Çalışmamıza benzer şekilde, Hint $[5,8-10]$ ve Kore $[11,12]$ popülasyonunda talus'ta Tip B oranı daha yaygındır. Bununla birlikte, Kore popülasyonunda Tip A'nın daha yaygın olduğunu bildiren [13] bir çalışma vardır (Tablo 4).

Tablo 4: Talus eklem yüzü tiplendirmesinin diğer çalışmalarla karşılaştırılması

\begin{tabular}{llccccccccc}
\hline Yazarlar & Popülasyon & TipA (\%) & $\begin{array}{c}\text { A1 } \\
\text { (\%) }\end{array}$ & $\begin{array}{c}\text { A2 } \\
\text { (\%) }\end{array}$ & $\begin{array}{c}\text { A3 } \\
\text { (\%) }\end{array}$ & $\begin{array}{c}\text { A4 } \\
(\%)\end{array}$ & $\begin{array}{c}\text { TipB } \\
\text { (\%) }\end{array}$ & $\begin{array}{c}\text { B1 } \\
\text { (\%) }\end{array}$ & $\begin{array}{c}\text { B2 } \\
\text { (\%) }\end{array}$ & $\begin{array}{c}\text { TipC } \\
\text { (\%) }\end{array}$ \\
\hline Arora ve ark. (8) & Hindistan & 19 & 3 & 0 & 0 & 16 & 79 & 78 & 1 & 2 \\
Bilodi (9) & Hindistan & 15 & 5 & 0 & 0 & 10 & 66,6 & 50 & 16,6 & 18,4 \\
Lee ve ark. (11) & Kore & 39,5 & 9,2 & 0 & 0 & 30,3 & 60,5 & 28,9 & 31,6 & 0 \\
Cho ve ark. (13) & Kore & 54,1 & 6,6 & 0 & 0 & 47,5 & 37,7 & 23 & 14,7 & 0 \\
Jung ve ark. (12) & Kore & 11 & - & - & - & - & 89 & 46,6 & 42,4 & 0 \\
Boyan ve ark. (6) & Türkiye & 1,7 & 0 & 1,7 & 0 & 0 & 98,3 & 55,9 & 42,4 & 0 \\
Prasad ve ark. (5) & Hindistan & 2,2 & 1,1 & 1,1 & 0 & 0 & 96,8 & 58,7 & 38,1 & 0 \\
Çalışmamız & Türkiye & 8,6 & 0 & 8,6 & 0 & 0 & 91,3 & 17,3 & 73,9 & 0 \\
\hline
\end{tabular}


Calcaneus'a ait eklem yüzlerinde de Hint, [4,7,14-20] Kore, [12] Afrika, [7] İspanya, [21] ve Amerika [22] popülasyonunda çalışmamızın sonuçlarına benzer şekilde Tip $B$ eklem yüzünün daha yaygın olduğu bildirilmiştir. Bizim çalışmamızdan farklı olarak Avrupa ve İngiltere popülasyonunda calcaneus Tip A oranı daha yüksek rapor edilmiştir [7]. Ayrıca çalışmamızın sonuçları daha önce Anadolu popülasyonunda yapılmış olan Uygur ve ark. [2] yaptığı çalışmanın sonuçlarına benzer olarak bulunmuştur [2]. Çalışmamızın sonuçları ile diğer çalışmaların sonuçları göz önüne alındığında hem talus hem de calcaneus'un eklem yüzü tiplerinde popülasyonlar arasında farklılıklar olduğu görülmektedir (Tablo 5).

Tablo 5: Calcaneus eklem yüzü tiplendirmesi'nin diğer çalışmalarla karşılaştırılması

\begin{tabular}{|c|c|c|c|c|c|c|c|c|c|c|}
\hline Yazarlar & Toplum & $\begin{array}{l}\text { TipA } \\
(\%)\end{array}$ & $\begin{array}{l}\text { A1 } \\
(\%)\end{array}$ & $\begin{array}{l}\text { A2 } \\
(\%)\end{array}$ & $\begin{array}{l}\text { A3 } \\
(\%)\end{array}$ & $\begin{array}{l}\text { A4 } \\
\text { (\%) }\end{array}$ & $\begin{array}{l}\text { TipB } \\
(\%)\end{array}$ & $\begin{array}{l}\text { B1 } \\
\text { (\%) }\end{array}$ & $\begin{array}{l}\text { B2 } \\
(\%)\end{array}$ & $\begin{array}{c}\text { TipC } \\
\text { (\%) }\end{array}$ \\
\hline Bunning ve Barnett (7) & Afrika & 36 & - & - & - & - & 63 & - & - & 0,8 \\
\hline Bunning ve Barnett (7) & İngiltere & 67 & - & - & - & - & 33 & - & - & 0 \\
\hline Bunning ve Barnett (7) & Hindistan & 17 & - & - & - & - & 61 & - & - & 0 \\
\hline Bunning ve Barnett (7) & Avrupa & 67 & - & - & - & - & 33 & - & - & 0 \\
\hline Campos ve Pellico (21) & İspanya & 46 & 3 & 21 & 16 & 6 & 54 & 29 & 25 & 0 \\
\hline $\begin{array}{l}\text { Uygur ve } \\
\text { ark. (2) }\end{array}$ & Türkiye & 39,4 & 4,1 & 13,1 & 17,2 & 5 & 58,4 & 25,4 & 33 & 2,2 \\
\hline Anjeneyulu ve ark. (19) & Hindistan & - & 10 & 10 & 11 & 5 & - & 43 & 19 & 24,1 \\
\hline $\begin{array}{l}\text { Sharada ve } \\
\text { ark. (17) }\end{array}$ & Hindistan & 28,6 & 5,33 & 13,66 & 9,66 & 3 & 67 & 50,3 & 16,6 & 1,33 \\
\hline $\begin{array}{l}\text { Jung ve } \\
\text { ark. (12) }\end{array}$ & Kore & - & 39 & 0 & 0 & 0 & - & 32 & 28,8 & 0 \\
\hline $\begin{array}{l}\text { Boyan ve } \\
\text { ark. (6) }\end{array}$ & Türkiye & - & 8,7 & 12,4 & 13,9 & 3,5 & - & 26,1 & 35,4 & 0 \\
\hline $\begin{array}{l}\text { Prasad ve } \\
\text { ark. (5) }\end{array}$ & Hindistan & 16,4 & 0 & 4,1 & 10,2 & 2,1 & 81,6 & 69,4 & 12,2 & 2,1 \\
\hline $\begin{array}{l}\text { Gupta ve } \\
\text { ark. (14) }\end{array}$ & Hindistan & 31 & 9 & 4 & 13 & 5 & 67 & 28 & 39 & 2 \\
\hline Padmanabhan (15) & Hindistan & 35 & - & - & - & - & 65 & - & - & 0 \\
\hline Mol ve ark. (16) & Hindistan & 26 & 22 & 2 & 2 & 0 & 74 & 40 & 34 & 0 \\
\hline $\begin{array}{l}\text { Sarvaiya ve } \\
\text { ark. (4) }\end{array}$ & Hindistan & 30,8 & 5,6 & 10,8 & 9,6 & 4,8 & 68,8 & 42,8 & 26 & 0,4 \\
\hline Garg ve ark. (18) & Hindistan & 25,8 & - & - & - & - & 72,3 & - & - & 1,6 \\
\hline Kullar ve ark. (20) & Hindistan & 27 & 15 & & 10,5 & 1,5 & 72,5 & 30 & 42,5 & 0,5 \\
\hline Ragab ve ark. (22) & Amerika & 37 & - & - & - & - & 45,6 & - & - & 0,2 \\
\hline Çalışmamız & Türkiye & 27,7 & 5,5 & 16,6 & 5,5 & 0 & 72,2 & 27,7 & 44,4 & 0 \\
\hline
\end{tabular}


Klinik tedavilerde, hekim tarafından talus ve calcaneus'un birlikte yapmış oldukları eklem yüzlerine ait toplumsal ve bireysel farklılıklar gösteren yaygın varyasyonel durumların, pes planus, pes ekinovarus, talocalcaneal artrit, talocalcaneal koalisyon, eklemi içeren kırıklar, valgus deformiteleri, subtalar instabilite ve subtalar implant uygulamaları gibi durumlarda etkili tedavi prosedürlerinin uygulanmasında bilinmesi önem arz etmektedir [2]. Tip B eklem yüzlerinin oluşturduğu eklemler daha fazla eklem hareketliliğine sahipken, Tip A eklem yüzlerinin oluşturduğu eklemler daha kısıtlı eklem hareketliliğine sahiptir. Ayrıca talocalcaneal artrite bağlı oluşan osteofit ve subkondral skleroz gibi artritik değişiklikler, Tip B grubuna ait calcaneuslarda Tip A grubuna kıyasla önemli ölçüde daha fazladır. Bunlar talus ile calcaneus arasındaki eklem stabilitesinin, birlikte oluşturdukları art. subtalaris ve bu kemiklerin art. talocalcaneonavicularis'e katılan eklem yüzü morfolojisine bağlı olduğunu göstermektedir [26].

Talus ve calcaneusun her ikisi için de Tip B ve Tip C eklem yüzüne sahip olan bireylerin ayak patolojilerinde egzersiz veya eklem mobilizasyonu gibi yumuşak doku teknikleriyle tedavi mümkün olabilecekken, Tip A eklem yüzüne sahip bireylerde basit tedavi teknikleri yetersiz kalacak ve daha komplike tedavi prosedürleri uygulanması gerekebilecektir [1].

Böhler açısının normal değerleri $25^{\circ}$ ila $40^{\circ}$ 'dir. Bu açı değerleri calcaneus'un kırılmasıyla azalmakta hatta negatif yönde değişebilmektedir. Yüksekten düşme gibi calcaneus'un ezildiği yaralanmaların çoğu anatomik konumundan dolayı facies articularis talaris posterior'u komprese ederek Böhler açısını azaltır. Bu nedenle Böhler açı değerleri üst sınırının kırıkların teşhisinde pratik bir önemi yokken alt sınır, kırıkların teşhisi için önemlidir. Özellikle normal tarafla karşılaştırmanın mümkün olmadığı bilateral calcaneus kırı̆ı̆ı olan bireylerde Böhler açısının bilinmesi önem arz etmektedir [23]. Ancak sağlıklı bireylerde Böhler açısının, sağ ve sol taraflar arasında $0^{\circ}$ ile $3^{\circ}$ arasında bir farklılık gösterebileceği unutulmamalıdır. Calcaneus kırıklarında, yapılan cerrahi işlem sonrası Böhler açısı normal değer aralığına getirilen hastaların postoperatif dönemde fonksiyonel sonuçlarının daha iyi olduğu bilinmektedir [24]. Bu nedenle Böhler açısı cerrahi işlem planlanmasında referans olarak kullanılabilir.

Böhler açısı farklı ırk ve toplumlarda farklı dağılım göstermektedir. Çalışmamızda belirlenen değerler $16^{\circ}$ ile $42^{\circ}$ arasında değişmektedir. $25^{\circ}$ alt sınır olarak kabul edilmekte ve çalışmamızda 4 adet $(\% 22,2)$ calcaneus'da Böhler açısı $25^{\circ}$ 'nin altında bulunmuştur (Tablo 2) Bu değerler Anadolu toplumu için referans değerlerin belirlenmesine katkıda bulunacaktır.

Ayrıca çalışmamızda calcaneus üzerinde yapılan ölçümlerden calcaneus anteroposterior uzunluğu ve transvers genişliği daha önce Anadolu ve Hindistan popülasyonlarında yapılan çalışmaların sonuçları ile benzerlik göstermektedir. [2,4,6,25]. Sulcus calcanei genişliği Anadolu ve [2,6] Kore popülasyonunda yapılan çalışmaların [12] sonuçları ile benzerlik göstermektedir. Sulcus calcanei uzunluğu ise daha önce yapıımış çalışmaların sonuçları ile farkıdır. Sebebi ise ölçümlerin alınmasında kullanılan farkı referans noktalarının yanı sıra ırksal farklılıklardan kaynaklanıyor olabilir (Tablo 6).

Tablo 6: Calcaneus'a ait morfometrik verilerin diğer çalışmalarla karşılaştırılması

\begin{tabular}{llcccc}
\hline Yazarlar & Toplum & $\begin{array}{c}\text { CAPU } \pm \text { Std. } \\
\text { Sapma (mm) }\end{array}$ & $\begin{array}{c}\text { CTG } \pm \text { Std. } \\
\text { Sapma (mm) }\end{array}$ & $\begin{array}{c}\text { SCG } \pm \text { Std. } \\
\text { Sapma (mm) }\end{array}$ & $\begin{array}{c}\text { SCU } \pm \text { Std. } \\
\text { Sapma (mm) }\end{array}$ \\
\hline Koshy ve ark. (25) & Hindistan & $73,6 \pm 5,7$ & $40,8 \pm 4,6$ & $21.1 \pm 2,4$ & $31,9 \pm 3,5$ \\
Uygur ve ark. (2) & Türkiye & $77,7 \pm 5,65$ & $47,5 \pm 4,2$ & $6,5 \pm 2,7$ & $30,4 \pm 3,1$ \\
Sarvaiya ve ark. (4) & Hindistan & $74,36 \pm 6,6$ & $38,57 \pm 3,12$ & $15,28 \pm 1,94$ & $10,44 \pm 1,66$ \\
Jung ve ark. (12) & Kore & - & - & $5.16 \pm 1,16$ & - \\
Boyan ve ark. (6) & Türkiye & $75,88 \pm 6,1$ & $44,88 \pm 4,09$ & $5,98 \pm 1,09$ & $32,13 \pm 2,99$ \\
Prasad ve ark. (5) & Hindistan & $75,27 \pm 11,24$ & $41,56 \pm 8,7$ & $5,66 \pm 3,51$ & $11,30 \pm 4,01$ \\
Çalışmamız & Türkiye & $75,49 \pm 6,00$ & $39,31 \pm 3,68$ & $5,72 \pm 1,73$ & $22,46 \pm 4,22$ \\
\hline
\end{tabular}


Çalışmamızda talus üzerinde yapılan ölçümlerden anteroposterior uzunluk ve transvers genişlik daha önce Anadolu ve Hint popülasyonlarında yapılan çalışmaların sonuçları ile benzerlik göstermektedir. $[2,4,6,25]$. Sulcus tali genişliği daha önce Anadolu popülasyonunda yapılan çalışmaların sonuçları ve Kore popülasyonunda yapılan çalışmanın [12] sonuçları ile benzerlik göstermektedir. Hindistan popülasyonunda Koshy ve ark. [25] yaptığı çalışmanın sonuçları ile farklılık göstermektedir. Sebebi ise ölçümlerin alınmasında kullanılan farklı referans noktalarının yanı sıra ırksal farklııklardan kaynaklanıyor olabilir (Tablo 7).

Tablo 7: Talus'a ait morfometrik verilerin diğer çalışmalarla karşılaştırılması

\begin{tabular}{llcccc}
\hline Yazarlar & Toplum & TAPU $(\mathbf{m m})$ & TTG $(\mathbf{m m})$ & STG $(\mathbf{m m})$ & STU (mm) \\
\hline Koshy ve ark. (25) & Hindistan & $52,8 \pm 5,8$ & $37,9 \pm 3,5$ & $27,8 \pm 4,4$ & $38 \pm 4,1$ \\
Lee ve ark. (11) & Kore & $53,9 \pm 3,2$ & $40,48 \pm 2,46$ & - & - \\
Jung ve ark. (12) & Kore & - & - & $4,76 \pm 1,0$ & - \\
Boyan ve ark. (6) & Türkiye & $51,78 \pm 4,09$ & $39,41 \pm 3,31$ & $5,65 \pm 1,57$ & $21,36 \pm 3,19$ \\
Prasad ve ark. (5) & Hindistan & $51,02 \pm 8,06$ & $36,61 \pm 5,65$ & $5,44 \pm 2,79$ & $11,41 \pm 5,78$ \\
Çalışmamız & Türkiye & $55,43 \pm 5,40$ & $41,49 \pm 3,95$ & $6,32 \pm 1,64$ & $22,14 \pm 3,29$ \\
\hline
\end{tabular}

Çalışmamızın bazı kısıtıııkları bulunmaktaydı. Bunlar incelediğimiz kemiklerin sayısının az olması, kemiklerin yaşının, cinsiyetinin ve kemik yapıyı etkileyecek metabolik patolojilerin (osteoporoz gibi) varlığının bilinmemesidir.

Çalışmamızda, Anadolu popülasyonunda daha önce yapılan çalışmalarla uyumlu bir şekilde talus ve calcaeus eklem yüzlerinin büyük oranda Tip B olduğunu belirledik. Bu bilgi Anadolu popülasyonunda çeşitli talocalcaneal düzeltme ameliyatlarında tedavi stratejilerinin planlanmasına yardımcı olacaktır. Popülasyonlar arası farklılıklar olmasından dolayı bu farklılıkların bilinmesinin ortopedik cerrahide ve antropolojik çalışmalarda önemli olacağını düşünüyoruz. Ayrıca farklı eklem yüzü tiplerine sahip olan sporculara ya da çalışanlara ait eklem yüzü tiplerinin iş ya da spora başlangıç öncesi taramalarda belirlenmesi, olası yaralanmaları önlemeyi ve maksimum performans elde etmeyi sağlayabilir. 


\section{Kaynakça}

[1] Bartoníček J, Rammelt S, Naňka O. Subtalar eklemin anatomisi, ayak ve ayak bileği klinikleri. 2018;23(3):315340.

[2] ]nUygur M, Atamaz F, Celik S, Pinar Y. The types of talar articular facets and morphometric measurements of the human calcaneus bone on Turkish race. Arch. Orthop. Trauma Surg. 2009;129(7):909-14.

[3] Işıklar ZU, Bilen FE. Kalkaneus kırıkları. TOTBID (Türk Ortopedi ve Travmatoloji Birliği Derneği) Dergisi. 2006;5(1-2):44-52.

[4] Sarvaiya BJ, Patel SV, Single G, Master DC. The types of talar articular facets and morphometric measurements of the human calcaneum bone of Gujarat region. Nat. J. Integr. Res. Med. 2012;3(3):34-8.

[5] Prasad SA, Rajasekhar SSSN. Morphometric analysis of talus and calcaneus. Surgical and Radiologic Anatomy. 2019;41(1):9-24.

[6] Boyan N, Ozsahın E, Kızılkanat E, Soames R, Oguz O. Morphometric Measurement and Types of Articular Facets on the Talus and Calcaneus in an Anatolıan Population. International Journal of Morphology. 2016;34(4).

[7] Bunning PSC, Barnett $\mathrm{CH}$. A comparison of adult and foetal talocalcaneal articulations. Journal of anatomy. 1965;99(1):71.

[8] Arora AK, Gupta SC, Gupta CD, Jeyasingh P. Variations in calcanean facets in Indian Tali. Anat. Anz. 1979;146(4):377-80.

[9] Bilodi AK. Study of calcaneal articular facets in human tali. Kathmandu Univ. Med. J. (KUMJ) 2006;4(1):75-7.

[10] Chavan SK, Satpute ST, Wabale RN. Pattern of talar articular facet of human calcaneum bone. Dent. Med. Sci., 2014;13(8):16-8.

[11] Lee JY, Jung MH, Lee JS, Choi BY, Cho BP. Types of calcaneal articular facets of the talus in Korean. Korean J. Phys. Anthropol. 2012;25(4):185-92.

[12] Jung MH, Choi BY, Lee JY, Han CS, Lee JS, Yang YC, Cho BP. Types of subtalar joint facets. Surg. Radiol. Anat. 2015;37(6):629-38.

[13] Cho HJ, Kwak DS, Kim IB. Analysis of movement axes of the ankle and subtalar joints: relationship with the articular surfaces of the talus. Proc. Inst. Mech. Eng. H, 2014;228(10):1053-8.

[14] Gupta SC, Gupta CD, Arora AK. Pattern of talar articular facets in Indian calcanei. J. Anat. 1977;124(3):6515.

[15] Padmanabhan R. The talar facets of the calcaneus an anatomical note. Anat. Anz. 1986;161(5):389-92.

[16] Mol PM, Silotry N, Kumari NH. Morphological study on patterns of talar articular facets of human calcanei. Int. J. Med. Clin. Res. 2012;3(3):136-9.

[17] Sharada R, Sneha K, Gupta C, Pai SR, Rairam GB. Nonmetrical study of the pattern of talar articular facets in South Indian dry calcanei. Surg. Radiol. Anat. 2012;34(6):487-91.

[18] Garg R, Dagal N, Kumar S, Shekhawat S. Study of patterns of talar articular facets of human calcanei and their clinical implications in population of Rajasthan. Indian J. Basic Appl. Med. Res. 2013;2(7):643-50.

[19] Anjaneyulu K, Philips C, Tamang BK, Kumar A. Patterns of talar articulating facets in adult human calcanei from North-East India and their clinical correlation. Asian J. Med. Sci. 2014;5(4):89-93.

[20] Kullar JS, Arora AK, Kapoor NS, Randhawa GK, Kullar KK. Morphology of talar articular facets of calcaneus and its clinical implications. Kashmir J. Med. Sci. 2015;1(1):10-4.

[21] Forriol Campos F, Gomez Pellico L. Talar articular facets (facies articulares talares) in human calcanei. Acta Anat. (Basel). 1989;134(2):124-7.

[22] Ragab AA, Stewart SL, Cooperman DR. Implications of subtalar joint anatomic variation in calcaneal lengthening osteotomy. J. Pediatr. Orthop. 2003;23(1):79-83. 
[23] Chen MY, Bohrer SP, Kelley TF. Boehler's angle: a reappraisal. Annals of emergency medicine. $1991 ; 20(2): 122-124$.

[24] Polat A, Demirtaş A, Azboy I, Uçar BY, Coşar Y, Gümüşsuyu G, Çakir IA. Kalkaneus kırıklarında kırık tipi ve açısal bozulmanın fonksiyonel sonuçlar üzerine etkisi. Dicle Tıp Dergisi. 2011;38(1)

[25] Koshy S, Vettivel S, Selvaraj KG. Estimation of length of calcaneum and talus from their bony markers. Forensic Sci. Int. 2002;129(3):200-4.

[26] Nemade KS, Meshram MM, Kasote AP, Kamdi NY. Arthritis of the subtalar joint associated with sustentaculum tali facet configuration. Int J Anat Res, (2014);2(4):684-88. 